\title{
EVALUACIÓN DEL ESTADO NUTRICIONAL, PATRONES DE CONSUMO ALIMENTARIO Y DE ACTIVIDAD FÍSICA EN ESCOLARES DEL CERCADO DE LIMA
}

\author{
Juan Pablo Aparco 1,2,a, William Bautista-Olórtegui1,a, Laura Astete-Robilliard ${ }^{1,3, a, c}$, Jenny Pillaca1,b
}

\begin{abstract}
RESUMEN
Objetivos. Evaluar el estado nutricional, los patrones alimentarios y de actividad física en escolares del Cercado de Lima. Materiales y métodos. Estudio descriptivo transversal. La muestra incluyó escolares del $1 .^{\circ}$ a $4 .^{\circ}$ grado de primaria de cuatro instituciones educativas públicas ubicadas en el Cercado de Lima. Las variables de estudio fueron el estado nutricional, nivel de hemoglobina, los patrones de consumo de alimentos y de actividad física. Se calcularon los porcentajes de las variables cualitativas y medidas de tendencia central para las cuantitativas, en el análisis bivariado se aplicó la prueba t y la prueba de chi cuadrado para analizar las diferencias según sexo. Resultados. De 824 escolares incluidos en el estudio, se encontró un $24 \%$ con obesidad, mientras que el sobrepeso afectó al $22 \%$, la proporción de talla baja fue de $5 \%$ y la de anemia fue $11,9 \%$, en todos los casos no hubo diferencias significativas según sexo. Más del $40 \%$ de escolares consumían 2 o más veces a la semana galletas saladas, jugos envasados y/o gaseosas. Además, el $28 \%$ de escolares eran sedentarios, encontrándose diferencias significativas según sexo $(p<0,05)$. Conclusiones. La obesidad es un problema vigente, con mayor frecuencia que el sobrepeso y que juntos afectan a casi el $50 \%$ de los escolares del estudio. Asimismo, se evidencia que existen patrones de sedentarismo y consumo frecuente de alimentos con alto contenido de azúcar, sal y/o grasas.
\end{abstract}

Palabras clave: Obesidad infantil; Escolares; Actividad física; Consumo de alimentos (fuente: DECS BIREME)

\section{ASSESSMENT OF THE NUTRITIONAL STATUS, PHYSICAL ACTIVITY, AND EATING HABITS OF SCHOOLCHILDREN IN CERCADO DE LIMA}

\begin{abstract}
Objectives. To assess the nutritional status, physical activity, and eating habits of schoolchildren in Cercado de Lima (Lima district). Materials and methods. Cross-sectional descriptive study. The sample included schoolchildren from first to fourth grade in four public elementary schools located in Cercado de Lima. The study variables were nutritional status, hemoglobin dose, physical activity, and eating habits. The percentages of the qualitative variables and central tendency measures for quantitative ones were calculated. The t-test and chi-squared test were applied to analyze differences between both genders. Results. Of 824 schoolchildren included in the study, $24 \%$ were obese, $22 \%$ were overweight, $5 \%$ had short stature, and $11.9 \%$ had anemia; in all instances, there were no substantial gender differences. More than $40 \%$ of schoolchildren would eat crackers and drink packaged juice and/or soda two or more times a week. In addition, $28 \%$ of schoolchildren were inactive; there were significant differences in both genders $(P<0.05)$. Conclusions. Obesity is an ongoing problem with higher rates than overweight and, together, the aforementioned problems affect almost $50 \%$ of schoolchildren surveyed. Similarly, the study revealed patterns associated with a sedentary lifestyle and frequent consumption of foods with high levels of sugar, salt, and/or fats.
\end{abstract}

Keywords: Childhood obesity; School children; Physical activity; Food consumption (source: MeSH NLM)

\section{INTRODUCCIÓN}

La obesidad infantil muestra una tendencia creciente a nivel mundial, el exceso de peso se ha incrementado en los últimos 30 años en un $47,1 \%$ en niños ${ }^{(1)}$. En el Perú, la prevalencia de obesidad en niños de 5 a 9 años ha aumentado de $7,7^{(2)}$ a 14,8\% ${ }^{(3)}$ en el periodo de 2007 a 2014.

Los efectos deletéreos de la obesidad infantil son múltiples, a nivel psicológico afecta la autoestima del niño y su esfera de relaciones sociales, personales, familiares y académicas ${ }^{(4)}$. A nivel biológico, la obesidad infantil se manifiesta a través de enfermedades cutáneas, ortopédicas, anormalidades lipídicas, apnea del sueño y otras enfermedades crónicas como: enfermedades cardiovasculares, diabetes mellitus e hipertensión, que condicionan un aumento del riesgo de mortalidad ${ }^{(5,6)}$. En el aspecto económico, las consecuencias de la obesidad como la discapacidad, la menor calidad de vida y la muerte temprana, en la población afectada, generan costos a la sociedad y al sistema de salud, que pueden variar entre 5 y $7 \%$ del total de los costes sanitarios de un país ${ }^{(7)}$.

\footnotetext{
Centro Nacional de Alimentación y Nutrición, Instituto Nacional de Salud. Lima, Perú.

Departamento Académico de Nutrición, Facultad de Medicina, Universidad Nacional Mayor de San Marcos. Lima, Perú.

Escuela de Nutrición, Universidad Peruana de Ciencias Aplicadas. Lima, Perú.

Nutricionista, ${ }^{\mathrm{b}}$ estadística, ${ }^{\mathrm{c}}$ magíster en Nutrición Pública.

Recibido: 04/04/2016 Aprobado: 24/08/2016
}

Citar como: Aparco JP, Bautista-Olórtegui W, Astete-Robilliard L, Pillaca J. Evaluación del estado nutricional, patrones de consumo alimentario y de actividad física en escolares del Cercado de Lima. Rev Peru Med Exp Salud Publica. 2016;33(4):633-9. doi: 10.17843/rpmesp.2016.334.2545 
Las intervenciones tempranas para prevenir la obesidad, sobre todo en el ámbito escolar, tienen potencial para ser una opción costo-efectiva frente a esta pandemia, ya que constituyen una buena oportunidad para formar hábitos alimentarios y de actividad física adecuados en los escolares ${ }^{(8)}$. Estas intervenciones serán efectivas si identifican y promueven conductas, acciones y prácticas concretas sobre el consumo de alimentos no saludables y sedentarismo; asimismo, deben apoyarse en teorías e investigación para elaborar las estrategias educativas que aborden las influencias y mediadores del cambio de conducta ${ }^{(9)}$.

A pesar de la necesidad de contar con evidencia sobre los patrones alimentarios y de actividad física para comprender más su rol como causas inmediatas del exceso de peso y diseñar modelos de intervención efectivos, en nuestro país predominan los estudios que solo brindan información acerca de la prevalencia de sobrepeso y obesidad en niños ${ }^{(2,3,10-12)}$.

Desde esa perspectiva, el objetivo del presente estudio fue evaluar el estado nutricional, patrones de consumo alimentario y de actividad física en escolares del $1 .^{\circ}$ al $4 .^{\circ}$ grado de primaria de instituciones públicas del Cercado de Lima, como línea de base para orientar los contenidos y actividades a realizar en la implementación de la intervención educativa motivacional para prevenir la obesidad infantil.

\section{MATERIALES Y MÉTODOS}

\section{DISEÑO DEL ESTUDIO}

Estudio descriptivo, transversal, que forma parte de una intervención educativa motivacional con diseño cuasiexperimental controlada y no aleatorizada. El estudio determinó, entre marzo y abril del 2014, el estado nutricional, patrones de consumo alimentario y de actividad física en escolares, como línea de base de la intervención motivacional.

\section{POBLACIÓN DE ESTUDIO}

Escolares de $1 .^{\circ}$ a $4 .^{\circ}$ grado de cuatro instituciones educativas públicas (IEP) de nivel primario y mixto (hombres y mujeres) de Lima Metropolitana. Se consideró incluir escolares de $1 .^{\circ}$ a $4^{\circ}$. grado porque en esta edad (6-10 años) se interiorizan los hábitos en los niños y se empieza a desarrollar los conceptos de salud y cuidado ${ }^{(8)}$. Además, se eligieron instituciones públicas porque concentran mayor cantidad de estudiantes, con menos recursos para afrontar posibles enfermedades crónicas y sus consecuencias a largo plazo.

\section{MUESTRA}

El tamaño de muestra se estimó para evaluar los efectos de la intervención motivacional, considerando una reducción de 0,3 desviaciones estándar en el promedio del puntaje $Z$ del IMC de los escolares intervenidos, y se ajustó por una tasa de rechazo del $25 \%$, considerando las posibles pérdidas del seguimiento durante el estudio y la varianza del indicador IMC $\left(s^{2}=1,36\right)$, calculado a partir de datos de niños de 6-10 años de la Encuesta Nacional de Hogares 2010, resultando una muestra de 316 niños para cada grupo de estudio. Dado que la intervención motivacional se asignó a nivel de IEP y se desarrolló en todos los escolares de $1 .^{\circ}$ a $4 .^{\circ}$ grado de primaria, la evaluación basal incluyó a todos estos escolares de las cuatro IEP ubicadas en el Cercado de Lima. Los criterios de inclusión fueron: pertenecer a una IEP de nivel primario y mixto, que no reciban beneficio del programa alimentario escolar Qali Warma ${ }^{(13)}$, además de la aprobación de los padres para participar en el estudio. Inicialmente se invitó a participar a 873 escolares, no se incluyeron a 49 de ellos, quedando finalmente 824 que completaron las encuestas socioeconómicas, de actividad física, de consumo de alimentos y datos antropométricos, solo 808 escolares completaron el dosaje de hemoglobina (Figura 1).

\section{PROCEDIMIENTOS}

Durante los meses de febrero y marzo de 2014 se visitó a los directores de las cuatro IEP para coordinar las actividades del estudio; simultáneamente, los encuestadores fueron capacitados durante 15 días, por especialistas del Centro Nacional de Alimentación y Nutrición (CENAN), en la aplicación de los formularios de recolección de datos y estandarizados en las técnicas de medición antropométrica y bioquímica según lo recomendado por el Instituto Nacional de Salud $(14,15)$. Inicialmente, se citó a los padres, a través de esquelas en los cuadernos de los escolares, a una reunión en la IEP donde se les explicó los detalles del estudio. Los padres que aceptaron participar fueron visitados, previa cita, en sus hogares donde se les aplico los cuestionarios. En el caso de padres que no acudieron a la reunión en la IEP se les visitó hasta en tres oportunidades en el hogar o centro laboral para coordinar una fecha para la entrevista. El trabajo de campo de los encuestadores se desarrolló por un periodo de 45 días, de lunes a domingo, de 5:00 am a 10:30 pm. La aplicación de los cuestionarios duraba 50 minutos aproximadamente. Al finalizar la entrevista se pesó y tomó la talla del niño; además, se le tomó una muestra de sangre capilar, la que fue examinada en el mismo lugar. Finalmente, se les entregó los resultados de antropometría y dosaje de hemoglobina a los padres, junto con orientación nutricional según el diagnóstico del niño, en caso de identificar desnutrición aguda, obesidad o anemia se refirió al niño al establecimiento de salud de la zona. Toda la visita en el hogar duraba aproximadamente 90 minutos.

\section{VARIABLES}

Antropométricas: se determinó el peso, la talla y el perímetro abdominal en todos los escolares, para ello se utilizaron balanzas electrónicas marca SECA® con una resolución de $0,1 \mathrm{~kg}$ y tallímetros de madera modelo OMS con una resolución de $0,1 \mathrm{~cm}$. Se calculó la prevalencia de desnutrición crónica: <-2 DE de Talla para edad, usando las tablas de referencia de OMS 
del 2006. Para determinar la prevalencia de niños con sobrepeso y obesidad se obtuvo el puntaje $Z$ del índice de masa corporal para edad (IMC) y se clasificó el estado nutricional utilizando los puntos de corte por edad de la OMS (16): <-3 DE, delgadez extrema; <-2 DE, bajo peso; entre -2 y $1 \mathrm{DE}$, normal; $>1 \mathrm{DE}$, sobrepeso y >2 DE, obesidad. La clasificación de los niveles de riesgo de perímetro abdominal se realizó considerando los puntos de corte propuestos por Fernández et al. ${ }^{(17)}$.

Dosaje de hemoglobina: para la evaluación de los niveles de hemoglobina, se utilizó el método de colorimetría en sangre capilar con el fotómetro portátil HemoCue ${ }^{\circledR}$. La prevalencia de anemia se determinó con los puntos de corte de $<11,5 \mathrm{~g} / \mathrm{dL}$ de hemoglobina, siguiendo la técnica establecida por el Instituto Nacional de Salud (15)

Patrones de consumo de alimentos: se obtuvo a través de un cuestionario de frecuencia de consumo de alimentos elaborado para el estudio y validado por expertos, se estructuró en base a una lista cerrada, constituida por 47 alimentos, bebidas y bocaditos clasificados en 13 grupos: a). Alimentos con cuatro grupos: frutas frescas, verduras frescas, leche y otros lácteos; b). Bocaditos con cuatro grupos: galletas dulces, galletas saladas, dulces (incluye caramelos, chicles, chupetines y gomitas) y chocolates (obleas, galletas con chocolate, wafer con chocolate); c). Preparaciones con dos grupos según donde se cocinó el alimento: frituras en casa y frituras fuera de casa; d). Bebidas con tres grupos: jugos bebidas envasadas (incluye néctar, jugo artificial de frutas $y$ chocolatada), gaseosas y agua sin azúcar (incluye agua hervida o embotellada). La frecuencia de consumo fue sistematizada en cinco categorías: Nunca, de 1 a 3 veces en el mes, 1 día en la semana, de 2 a 4 días a la semana y de 5 a 7 días a la semana. Para determinar la frecuencia de consumo adecuado de alimentos se utilizaron las recomendaciones de la Sociedad Española de Nutrición Comunitaria (SENC) para niños y adolescentes ${ }^{(18)}$.

Patrones de actividad física: para evaluar los patrones de actividad física se empleó un cuestionario de actividad física elaborado por el Instituto de Nutrición y Tecnología de Alimentos (INTA) de Chile, que ha sido validado en comparación con acelerómetros triaxiales como patrón de oro (19); asimismo, este cuestionario ha sido utilizado por Liria et al. en un estudio de escolares de Lima y Callao ${ }^{(20)}$. El instrumento tiene cinco categorías: a). Horas diarias durmiendo/acostado; b). horas diarias de actividad mínima (actividades que se pueden realizar sentado, como atender la clase, viajar en bus, leer, ver televisión, usar la computadora, videojuegos, comer, etc.); c). número de cuadras caminadas diariamente; d). horas diarias de juegos recreativos, y e). horas semanales de ejercicios o deportes programados. La calificación de actividad física se realizó utilizando los puntajes propuestos por Liria et al., resultando en nivel bueno: de 7 a 10 puntos, regular: de 4 a 6 puntos y malo: menor o igual a 3 puntos.

\section{ANÁLISIS ESTADÍSTICO}

Se realizó un análisis descriptivo mediante distribuciones de frecuencias y porcentajes para las variables cualitativas y medidas de tendencia central (media \pm desviación estándar) para las variables cuantitativas. Se evalúo la normalidad de la distribución de las variables principales con la prueba de Shapiro-Wilk. Luego se realizó un análisis bivariado para comparar el estado nutricional, a través de sus diferentes indicadores, y el nivel de actividad física, según sexo. En el caso de variables cuantitativas numéricas, para determinar si hubo diferencia significativa entre hombres y mujeres se utilizó la prueba de $\mathrm{t}$ de Student para muestras independientes. Para las variables categóricas se empleó la prueba de chi cuadrado, en el caso del IMC se utilizó la prueba exacta de Fisher para corregir la estimación. En todos los casos se consideró un $p<0,05$ como nivel de significación estadística y los análisis estadísticos se realizaron mediante el paquete estadístico STATA/SE versión 12.0.

\section{ASPECTOS ÉTICOS}

El protocolo de estudio fue aprobado por el Comité Institucional de Ética en Investigación del INS. Antes de iniciar el estudio se coordinó con los representantes de cada institución educativa y para la recolección de datos y toma de muestras se solicitó el consentimiento informado a ambos padres, explicando los alcances de la investigación.

\section{RESULTADOS}

Se obtuvo información de 824 escolares de 6 y 12 años de edad, el $56,9 \%$ de los escolares fueron hombres, el $45 \%$ de niños no tenía ningún seguro médico, al analizar las características de los escolares según sexo, solo resultó significativa la diferencia del promedio de puntaje $Z$ del índice de masa corporal por edad $(p<0,05)$, siendo mayor en varones (Tabla 1).

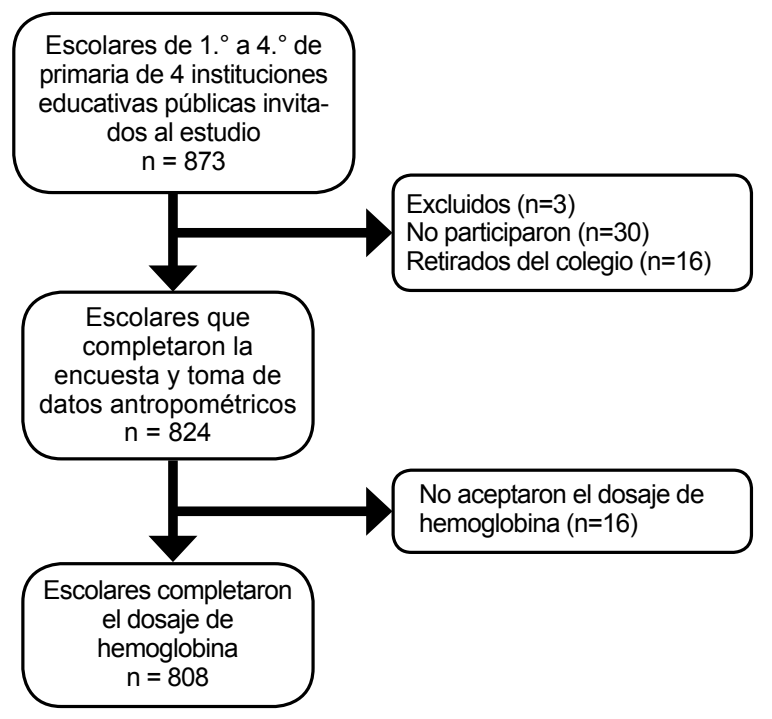

Figura 1. Flujograma de la muestra 
Tabla 1. Características generales de los escolares del Cercado de Lima, 2014, según sexo.

\begin{tabular}{lcccc}
\hline \multirow{2}{*}{ Características } & Total & Hombres & Mujeres & \multirow{2}{*}{ valor $\boldsymbol{p}^{*}$} \\
\cline { 2 - 3 } & Media (DE) & Media (DE) & Media (DE) & 0,581 \\
\hline Edad (años) & $7,52(1,2)$ & $7,54(1,2)$ & $7,51(1,2)$ & 0,350 \\
\hline Peso (Kg) & $28,7(8,2)$ & $28,7(8,1)$ & $28,6(8,1)$ & 0,063 \\
Talla (cm) & $124,4(8,6)$ & $124,4(8,3)$ & $124,3(9,0)$ & 0,031 \\
Puntaje Z IMC (BAZ) & $1,01(1,3)$ & $1,1(1,3)$ & $0,91(1,2)$ & 0,513 \\
Puntaje Z Peso/Edad (WAZ) & $0,50(1,3)$ & $0,51(1,3)$ & $0,49(1,3)$ & 0,871 \\
Puntaje Z Talla/Edad (HAZ) & $-0,45(0,9)$ & $-0,47(0,9)$ & $-0,41(0,9)$ & 0,403 \\
Hemoglobina (g/dL) & $12,5(0,9)$ & $12,5(0,8)$ & $12,5(0,9)$ & 0,780 \\
\hline Perímetro abdominal (cm) & $62,5(8,9)$ & $62,8(8,8)$ & $62,1(8,7)$ & \\
\hline
\end{tabular}

*Prueba t de Student

El porcentaje de obesidad en escolares fue de $24 \%$ y de sobrepeso fue de $22 \%$. En base al perímetro abdominal el $21 \%$ de los escolares tenía riesgo alto de padecer alguna enfermedad metabólica. Asimismo, la proporción de escolares con talla baja alcanzó un $5 \%$ mientras que el $11 \%$ sufría de anemia, en ningún caso se encontraron diferencias significativas según sexo (Tabla 2).

Respecto a los patrones de consumo de alimentos, el $60 \%$ de escolares consume frutas frescas casi a diario (entre 5 a 7 días a la semana), mientras que el $35 \%$ lo consume en forma interdiaria. El consumo de verduras frescas refleja un patrón preocupante, pues solo el $18 \%$ de escolares cumple la recomendación de consumirlas a diario, incluso un $12 \%$ refiere que nunca las ha probado. El $68 \%$ de escolares manifiesta que consumen frituras en casa 2 o más veces a la semana, mientras que el $52 \%$ consume galletas en la misma frecuencia. Además, el $40 \%$ de niños consume jugos envasados entre dos a cuatro veces por semana y un $9 \%$ casi diariamente, mientras que un $12 \%$ consume gaseosas casi a diario (Tabla 3 ).

La evaluación de los patrones de actividad física durante los días de clases (lunes a viernes), indican que dormir (cerca de nueve horas y media/día) y realizar actividades mínimas (diez horas/día) demandan el mayor tiempo durante el día. Asimismo, permanecer frente a alguna pantalla (TV, videojuegos, computadora, etc.) consume en promedio 117 minutos al día y para realizar juegos

Tabla 2. Estado nutricional de los escolares del Cercado de Lima, 2014, según sexo

\begin{tabular}{|c|c|c|c|c|}
\hline \multirow{2}{*}{ Estado nutricional } & Total & Hombres & Mujeres & \\
\hline & $\%(n)$ & $\%(n)$ & $\%(n)$ & Valor $p$ \\
\hline Índice de masa corporal & & & & $0,331^{*}$ \\
\hline Delgadez & $0,6(5)$ & $0,4(2)$ & $0,8(3)$ & \\
\hline Normal & $52,7(434)$ & $51,6(242)$ & $54,1(192)$ & \\
\hline Sobrepeso & $22,5(185)$ & $21,6(101)$ & $23,7(84)$ & \\
\hline Obesidad & $24,2(200)$ & $26,4(124)$ & $21,4(76)$ & \\
\hline Perímetro abdominal & & & & 0,473 \\
\hline Bajo riesgo & $59,1(486)$ & $58,2(272)$ & $60,3(214)$ & \\
\hline Riesgo alto & $21,1(174)$ & $22,6(106)$ & $19,2(68)$ & \\
\hline Riesgo muy alto & $19,8(163)$ & $19,2(90)$ & $20,5(73)$ & \\
\hline Talla baja & & & & 0,421 \\
\hline Sí & $5,2(43)$ & $5,8(27)$ & $4,5(16)$ & \\
\hline No & $94,8(781)$ & $94,2(442)$ & $95,5(339)$ & \\
\hline Anemia & & & & 0,180 \\
\hline Sí & $11,9(96)$ & $10,6(49)$ & $13,6(47)$ & \\
\hline No & $88,1(712)$ & $89,4(414)$ & $86,4(298)$ & \\
\hline
\end{tabular}

* Prueba exacta de Fisher 
Tabla 3. Frecuencia de consumo de alimentos en escolares del Cercado de Lima, 2014

\begin{tabular}{lccccc}
\hline & Nunca & $\begin{array}{c}\mathbf{1 - 3} \text { veces al } \\
\text { mes }\end{array}$ & $\begin{array}{c}\text { 1 día a la } \\
\text { semana }\end{array}$ & $\begin{array}{c}\text { 2-4 días a la } \\
\text { semana }\end{array}$ & $\begin{array}{c}\text { 5-7 días a la } \\
\text { semana }\end{array}$ \\
\hline Alimentos & $\%(\mathbf{n})$ & $\%(\mathbf{n})$ & $\%(\mathbf{n})$ & $\%(\mathbf{n})$ & $\%(\mathbf{n})$ \\
\hline Frutas frescas & $0,6(5)$ & $1,6(13)$ & $3,0(25)$ & $35,2(290)$ & $59,6(491)$ \\
Verduras frescas & $11,8(97)$ & $8,6(72)$ & $12,9(106)$ & $48,7(401)$ & $18,0(148)$ \\
\hline Leche & $2,2(18)$ & $2,8(23)$ & $3,2(26)$ & $29,6(244)$ & $62,2(513)$ \\
\hline Otros lácteos: yogur, queso & $0,8(7)$ & $4,1(34)$ & $9,8(81)$ & $55,0(453)$ & $30,3(294)$ \\
\hline Frituras en casa & $2,5(21)$ & $8,1(67)$ & $21,1(174)$ & $58,3(480)$ & $10,0(82)$ \\
Frituras fuera de casa & $7,3(60)$ & $44,7(368)$ & $27,4(226)$ & $17,7(146)$ & $2,9(24)$ \\
\hline Galletas saladas & $5,3(44)$ & $20,7(171)$ & $21,4(176)$ & $41,1(339)$ & $11,5(94)$ \\
Galletas dulces & $10,8(89)$ & $28,5(235)$ & $21,7(179)$ & $30,2(249)$ & $8,8(72)$ \\
\hline Dulces & $11,8(97)$ & $39,6(327)$ & $18,6(153)$ & $22,6(186)$ & $7,4(61)$ \\
Chocolates, bombones & $21,5(177)$ & $42,7(352)$ & $19,8(163)$ & $13,1(108)$ & $2,9(24)$ \\
\hline Jugos envasados & $12,7(105)$ & $23,7(195)$ & $15,0(124)$ & $39,1(322)$ & $9,5(78)$ \\
\hline Gaseosas & $7,4(61)$ & $28,2(232)$ & $22,3(184)$ & $30,0(247)$ & $12,1(100)$ \\
\hline Agua sin azúcar & $9,5(78)$ & $3,2(27)$ & $4,9(40)$ & $15,4(127)$ & $67,0(552)$ \\
\hline
\end{tabular}

recreativos se destinan en promedio $70 \mathrm{~min}$ al día, con diferencia significativa según sexo $(p<0,01)$ (Tabla 4).

Solo un $8 \%$ de los escolares presentó un buen nivel de actividad física, en contraste con el $28 \%$ que tenía un nivel malo (sedentarios); con diferencias significativas entre hombres y mujeres, destacando que las mujeres tienen mayor nivel de sedentarismo (Tabla 5).

\section{DISCUSIÓN}

Los resultados del estudio muestran que cerca de la mitad de los escolares del estudio tienen exceso de peso y que el $40 \%$ presentan algún nivel de riesgo de desarrollar enfermedades metabólicas debido a la acumulación central de la grasa en el abdomen. Proporciones similares de sobrepeso y obesidad en escolares de Lima han sido reportados por Tarqui-Mamani et al. ${ }^{(3)}$, RosadoCipriano et al. ${ }^{(12)}$ y Gago et al. (21) en Villa el Salvador. Estas cifras sugieren que el problema de obesidad en escolares está vigente, en especial en zonas urbanas como Lima Metropolitana ${ }^{(3)}$.
La frecuencia de obesidad fue mayor que el sobrepeso, esto podría deberse a que el aumento de peso está afectando progresivamente a niños cada vez más pequeños, la evidencia científica muestra que existen periodos sensibles en que el entorno programa y desarrolla obesidad en niños ${ }^{(22)}$, con una aparición temprana de adiposidad ${ }^{(23)}$ de tal forma que a los seis y siete años encontramos niños obesos que ya tuvieron sobrepeso. Este patrón inusual de predominancia de la obesidad también ha sido reportado en estudios similares realizados en Lima ${ }^{(3,12)}$ y prevalencias de obesidad iguales o mayores que el sobrepeso en escolares han sido reportadas en Brasil ${ }^{(24)}$ y México ${ }^{(25)}$.

Los patrones de consumo de alimentos muestran que la mayoría de escolares tienen un consumo de 2 o más veces a la semana de alimentos con alto contenido de sal, azúcar y/o grasas, resultados similares se han reportado en México ${ }^{(25)}$ y España ${ }^{(26)}$, donde los alumnos usuarios de comedores escolares consumían entre 2 a 3 veces a la semana galletas, chocolates, snacks salados, zumo comercial y refrescos (gaseosas).

Tabla 4. Tiempo promedio de actividad física en escolares del Cercado de Lima, 2014, según sexo

\begin{tabular}{|c|c|c|c|c|}
\hline \multirow{2}{*}{ Tiempo } & Total & \multicolumn{3}{|l|}{ Hombres } \\
\hline & Media (DE) & Media (DE) & Media (DE) & valor $p^{*}$ \\
\hline Durmiendo/acostado (min/día) & $567,33(66,12)$ & $567,58(67,69)$ & $567,01(64,09)$ & 0,90 \\
\hline Actividad mínima (min/día) & $602,34(124,18)$ & $601,88(138,62)$ & $602,94(102,22)$ & 0,90 \\
\hline Sentado frente a pantallas (min/día) & $117,67(71,78)$ & $119,75(73,64)$ & $114,93(69,24)$ & 0,33 \\
\hline Juegos recreativos (min/día) & $70,02(64,49)$ & $77,71(69,87)$ & $59,86(55,07)$ & $<0,01$ \\
\hline Deportes programados (min/semana) & $127,40(70,97)$ & $128,73(72,22)$ & $125,64(69,33)$ & 0,53 \\
\hline
\end{tabular}

${ }^{*} \mathrm{t}$ de student para muestras independientes 
Tabla 5. Clasificación del nivel de actividad física en escolares del Cercado de Lima, 2014, según sexo

\begin{tabular}{lcccc}
\hline & Total & Hombres & Mujeres & \\
\cline { 2 - 4 } $\begin{array}{l}\text { Nivel de } \\
\text { actividad } \\
\text { física }\end{array}$ & $\%(\mathbf{n})$ & $\%(\mathrm{n})$ & $\%(\mathbf{n})$ & $\begin{array}{c}\text { Valor } \\
\boldsymbol{p}\end{array}$ \\
\hline Malo & $28,0(231)$ & $24,3(114)$ & $33,0(117)$ & $0,007^{*}$ \\
\hline Regular & $63,9(526)$ & $65,9(309)$ & $61,1(217)$ & \\
Bueno & $8,1(67)$ & $9,8(46)$ & $5,9(21)$ & \\
\hline
\end{tabular}

† Prueba de chi cuadrado

En cuanto a los patrones de actividad física encontramos que los resultados difieren de lo reportado por Liria et al. (20) quienes encontraron que menos del $1 \%$ de escolares tenía un buen nivel de actividad física, al respecto debemos considerar que las poblaciones de estudio no son las mismas, en el presente estudio se evaluaron niños de $1 .^{\circ}$ a $4 .^{\circ}$ de primaria, mientras que el estudio de Liria se incluyó escolares de $3 .^{\circ}$ a $6 .^{\circ}$ de primaria, la evidencia muestra que la actividad física en escolares se reduce conforme aumenta la edad y los grados de estudios ${ }^{(25)}$. En ambos casos, los resultados muestran que la mayoría de escolares tienen un nivel de actividad física considerado de malo a regular.

La obesidad es un problema multicausal que incluye factores etiológicos que aparecen incluso desde el embarazo y que, dependiendo del tiempo y la compleja interacción de las múltiples exposiciones, se desencadenaría tarde o temprano. No existen muchos estudios sobre las causas de obesidad en escolares, los disponibles muestran que la densidad calórica de la dieta, más que los patrones de alimentación, están relacionados con el peso corporal en niños y adolescentes. Una revisión sistemática realizada en países en desarrollo encontró que la obesidad está inversamente asociada al nivel socioeconómico, en parte, porque los alimentos más baratos son los que más densidad calórica presentan ${ }^{(27)}$, sin embargo, en países desarrollados los niños con mayor riesgo de obesidad son aquellos cuyos padres son de menor nivel socioeconómico o educativo ${ }^{(28)}$. Como opera el nivel socioeconómico en cada contexto, es una pregunta que necesita abordarse para ajustar las intervenciones. En nuestro estudio encontramos que, si bien un $92 \%$ de niños tomaba desayuno diariamente, cerca del $62 \%$ lo compraba en la calle lo que implica consumir preparaciones con mayor densidad energética.

Una de las limitaciones del estudio es que se realizó en cuatro instituciones educativas públicas de un distrito de
Lima, por lo que sus resultados no se pueden inferir a un mayor nivel; sin embargo, las estimaciones de otros estudios como el de Tarqui-Mamani et al. (3) que usa la Encuesta Nacional de Hogares (ENAHO), encontró proporciones similares de sobrepeso y obesidad en Lima Metropolitana. Asimismo, otro aspecto limitante fue que el consumo de alimentos solo se recogió de manera cualitativa, por lo que no se puede cuantificar el aporte o peso de cada uno de los alimentos consumidos. Así, el cuestionario de frecuencia de consumo de alimentos brinda información que permite recoger los patrones o hábitos alimentarios con la finalidad de proponer conductas clave a modificar y establecer metas de cambio ${ }^{(29)}$. La medición de la actividad física también tuvo limitaciones, ya que el cuestionario utilizado se basa en información referida por la madre y se aplica solo para cinco días de la semana. La evidencia muestra que los métodos subjetivos de valoración de actividad física pueden tener errores en la estimación para cuantificar el gasto energético por actividad física; sin embargo, en el estudio se aplicó un test de actividad física validado con acelerómetros ${ }^{(17,18)}$, además, se ha descrito que los escolares tienen patrones homogéneos del uso de su tiempo, por lo que se puede recoger a través de cuestionarios de manera fiable ${ }^{(30)}$.

En conclusión, los resultados del estudio muestran que la obesidad es un problema de salud vigente, que se presenta con mayor frecuencia que el sobrepeso y que juntos afectan a casi el $50 \%$ de los escolares que participaron en el estudio. Asimismo, se evidencia que existen patrones de actividad física y de consumo de alimentos en formas inadecuadas, lo que se refleja en que casi un tercio de escolares son sedentarios y en un consumo frecuente mayor a lo recomendado de frituras, galletas saladas, jugos envasados y gaseosas.

Los resultados del estudio permitieron elaborar un diagnóstico más preciso de la realidad y sirvieron como eje para diseñar el modelo de la intervención motivacional y los mensajes clave para promover conductas y prácticas saludables en los niños.

Contribuciones de autoría: JPA, WB y LA han participado en la concepción y diseño del artículo. JPA, WB y JP participaron en la recolección de datos. JPA, LA y JP realizaron el análisis de datos. Todos los autores participaron en la redacción del borrador del artículo, lo revisaron y aprobaron la versión final.

Fuentes de financiamiento: el proyecto fue financiado por el Instituto Nacional de Salud de Perú.

Conflictos de interés: los autores declaran no tener conflictos de interés. 


\section{REFERENCIAS BIBLIOGRÁFICAS}

1. $\mathrm{Ng} \mathrm{M}$, Fleming $\mathrm{T}$, Robinson $\mathrm{M}$, Thomson B, Graetz N, Margono C, et al. Global, regional, and national prevalence of overweight and obesity in children and adults during 1980-2013: a systematic analysis for the Global Burden of Disease Study 2013. Lancet [Internet]. 2014;384(9945):766-81.

2. Tarqui-Mamani C, Sánchez-Abanto J, Álvarez-Dongo D, Gómez-Guizado G, Valdivia-Zapana S. Tendencia del sobrepeso, obesidad y exceso de peso en el Perú. Rev peru epidemiol. 2013;17(3):1-7.

3. Tarqui-Mamani C, Alvarez-Dongo D: Estado Nutricional de la Población por Etapas de Vida; 2013-2014. Informe Técnico. Instituto Nacional de Salud. Lima, 2015.

4. Ebbeling CB, Pawlak DB, Ludwig DS. Childhood obesity: public-health crisis, common sense cure. Lancet. 2002;360(9331):473-82.

5. Freedman DS, Patel DA, Srinivasan SR, et al. The contribution of childhood obesity to adult carotid intima-media thickness: the Bogalusa Heart Study. Int J Obes (Lond). 2008; 32(5):749-56.

6. Han JC, Lawlor DA, Kimm SY. Childhood obesity. Lancet. 2010 May 15;375(9727):1737-48.

7. John J, Wolfenstetter SB, Wenig CM. An economic perspective on childhood obesity: recent findings on cost of illness and cost effectiveness of interventions. Nutrition 2012; 28: 829-39.

8. Pérez-Rodrigo C, Aranceta J. Schoolbased nutrition education: lessons learned and new perspectives. Public Health Nutr. 2001;4(1A):131-9.

9. Contento IR. Nutrition education: linking research, theory, and practice. Asia Pac J Clin Nutr. 2008;17 Suppl 1:176-9.

10. Pajuelo-Ramirez J, Miranda-Cuadros M, Campos-Sanchez M, Sanchez-Abanto J, José: Prevalencia de sobrepeso y obesidad en niños menores de cinco años en el Perú 2007-2010. Rev Peru Med Exp Salud Publica. 2011; 28(2): 222-27.

11. Lozano-Rojas G, Cabello-Morales E, Hernádez-Diaz $\mathrm{H}$, Loza-Munarriz C. Prevalencia de sobrepeso y obesidad en adolescentes de un distrito urbano de Lima, Perú 2012. Rev Peru Med Exp Salud Publica. 2014;31(3):494-500.

12. Rosado-Cipriano M, Silvera-Robles VL, Calderón-Ticona JR. Prevalencia de sobrepeso y obesidad en niños escolares. Rev Soc Peru Med Interna. 2011;24(4):163-9.

13. Qali Warma: Programa Nacional de Alimentación [Internet] Ministerio de Desarrollo e Inclusión Scocial (MIDIS): [Consultado el 17 de octubre del 2016]. Disponible en: http://www.qw.gob. pe/?page_id $=2$.

14. Instituto Nacional de Salud (INS), Centro Nacional de Alimentación y Nutrición. La medición de la talla y el peso. Guía para el personal de salud del primer nivel de atención. Lima: INS; 2004.

15. Instituto Nacional de Salud (INS), Centro Nacional de Alimentación y Nutrición. Procedimiento para la determinación de hemoglobina mediante hemoglobinómetro portátil. Lima: INS; 2013.

16. WHO: The WHO Child Growth Standars 2006 BMI for age [citado el 23 de febrero del 2016]. Disponible en: http://www.who.int/childgrowth/ standards/bmi_for_age/en/index.html.

17. Fernández JR, Redden DT, Pietrobelli A, Allison DB. Waist circumference percentiles in nationally representative samples of African-American, EuropeanAmerican, and Mexican-American children and adolescents. J Pediatr. 2004; 145(4):439-44.

18. Serra Majem L., Aranceta J, Mataix J. Documento de consenso. Guías alimentarias para la población española. SENC. Barcelona: SG editores; 1995. p. 301-8.

19. Godard MC, Rodríguez NM, Díaz N, Lera L, Salazar G, Burrows R. Valor de un test clínico para evaluar actividad física en niños. Rev. Méd. Chile. 2008; 136(9): 1155-1162.

20. Liria MR, Mispireta ML, Lanata CF, Creed-Kanashiro HM. Perfil Nutricional en escolares de Lima y Callao. Lima: Instituto de Investigacion Nutricional, 2008.

21. Gago J, Rosas $O$, Huayna M, Jiménez D, Córdova F, Navarro A, et al. Efectividad de una intervención multisectorial en educación alimentaria nutricional para prevenir y controlar el sobrepeso y la obesidad en escolares de cuatro instituciones educativas públicas del distrito de Villa El Salvador. Rev peru epidemiol. 2014; 18 (3): e04.

22. Dietz WH. Critical periods in childhood for the development of obesity. Am J Clin Nutr. 1994 May;59(5):955-9.

23. Rolland-Cachera MF, Deheeger M, Maillot M, Bellisle F. Early adiposity rebound: causes and consequences for obesity in children and adults. Int J Obes (Lond). 2006;30 Suppl 4:S11-7. Erratum in: Int J Obes (Lond). 2010;34(7):1230.

24. Instituto Brasileiro de Geografia e Estatistica (IBGE). Pesquisa de Orçamentos Familiares 2008-2009 Antropometria e Estado nutricional de Crianças, Adolescentes e Adultos no Brasil: IBGE, Rio de Janeiro, 2009

25. Ogden CL, Connor S, Rivera JA, Caroll M,Shields M, Flegal K. The epidemiology of childhood obesity in Canada, México and the United States. In: Moreno LA, Pigeot I, Ahrens W. Epidemiology of Obesity in Children and Adolescents: Prevalence and Etiology. Ed. Springer Verlag, 2010.

26. Aranceta J, Pérez C, Serra L, Delgado A. Hábitos alimentarios de los alumnos usuarios de comedores escolares en España. Estudio "Dime Cómo Comes". Aten Prim. 2004;33(3):131-9.

27. Pérez-Escamilla R, Obbagy JE, Altman JM, Essery EV, McGrane MM, Wong YP, et al. Dietary energy density and body weight in adults and children: a systematic review. J Acad Nutr Diet. 2012 May;112(5):671-84. doi: 10.1016/j. jand.2012.01.020

28. Dinsa GD, Goryakin Y, Fumagalli E, Suhrcke M. Obesity and socioeconomic status in developing countries: asystematic review. Obes Rev [Internet]. 2012 Nov [cited 2016 Jun 30];13(11):1067-79.

29. Barriuso L, Miqueleiz E, Albaladejo R, Villanueva R, Santos JM, Regidor E. Socioeconomic position and childhoodadolescent weight status in rich countries: a systematic review, 1990-2013. BMC Pediatr [Internet]. 2015 [cited 2016 Jun 30];15:129.

30. Walter Willett. Nutritional Epidemiology, Third Edition. Oxford University Press, 2012.

31. Hernández B, Gortmaker SL, Laird NM, Colditz GA, Parra-Cabrera S, Peterson KE. Validity and reproducibility of a physical activity and inactivity questionnaire for Mexico City's schoolchildren. Salud Publica Mex 2000;42:315-323.

Correspondencia: Juan Pablo Aparco Balboa Dirección: Jr. Tizón y Bueno 276, Jesús María. Lima, Perú

Teléfono: (+51): 748-0000 anexo 6626 Correo electrónico: japarco@ins.gob.pe 